\title{
(Supplementary) Neutrophil Function in the Infection on Compromised Host
}

\author{
Eiro Tsubura, MD \\ The Third Department of Internal Medicine, School of Medicine, \\ The University of Tokushima, Tokushima
}

Regardless of a spread use of newly developed antibiotic chemotherapy against infections disease, infection occurred in a compromised host is usually hard to cure. It is important to know which factors are concerning in these infection for their treatment and prevention.

As the results of our observation, secondary infection in compromised states of malignancy was high incidence in acture leukemia, malignant lymphoma and various cancer. There are many complicated host defence factors influencing these infections, however, the most obvious factor was the decrease of the absolute number of neutrophil after intensive antineoplastic chemotherapy in the leukemic patients. On the other hand suppressed cell-mediated immunity was seemed to partly related in the occurrence of infections in the cancer patients.
The depressed neutrophilic functions was also denoted in the compromised states but the accurate relationship between a cause of infection and depressed functions of neutrophil was difficult to determine.

It is necessary to establish a practical parameter of phagocytic and intracellular killing activities by neutrophil for early detection of compromised states during a course of malignancy in order to prevent secondary infection in these patients.

It is also necessary to find out some augmenting agents which modulate or promote host defence activity against infection. We tested and screened several augmenting agents such as Nocardis rubra cell wall skelton and bacterial product in the compromised mice infected with Escherichia coli.

\section{Prevention and Treatment of Conditions Liable to Microbial Infections}

\author{
Yuichi SHIOKAwA, MD \\ Juntendo University School of Medicine, \\ Department of Rheumatology, Tokyo
}

Invasion of microorganisms into the human body is first prevented by a variety of natural barriers, e.g. the skin, mucous membranes of the GI and respiratory tract etc. If these barriers are penetrated by the organisms, then immune and non-immune mechanisms will endeavor to hinder the harmful influences of microbes, including endo- and exotoxins. Thus, the defense system of the host against infections is efficient, but very complicated, and the details concerning it are not fully clarified.

\section{Infectability by Microbes}

It is well known that immunological diseases, e.g. systemic lupus erythematosus (SLE) and rheumatoid arthritis (RA) are frequently accompanied by infections. The infectability seen in these disorders is due to the depressed functions in lymphocytes, macrophages and other cells of the reticuloendothelial system. The use of adrenocorticosteroids, immunosuppressants and other drugs also contributes to this condition.

In my hospital, 307 patients with SLE have 
been observed, among which 95 (30.9\%) have had infections. The organs involved were respiratory in $25(8.1 \%)$, urinary in $20(6.5 \%)$, digestive in $12(3.9 \%)$, septicemic in $7(2.3 \%)$, meningeal in $5(1.6 \%)$, etc. The causative organisms were, Herpes virus in $15(15.8 \%)$, E. coli in 12 (12.6\%); Candida albicans in 11 (11.6\%); Cryptococcus neoformans in 3 (3.2\%); Aspergillus fumigatus, Mycobacterium tuberculosis and Salmonella typhi murium in 2 each $(2.1 \%)$; and Pneumocystis carinii, Mycoplasma pneumoniae and Papova virus in one each $(1.1 \%)$. The liability to infections is usually a prevailing cause of death in this disease. In patients with SLE who we have followed up, 53 out of 305 (17.3\%) died. The causes of death were uremia $(10.7 \%)$ and the central nervous system syndrome $(3.9 \%)$, followed by infections $(3.6 \%)$ which we are discussing here, and others.

It was noted that in SLE, in addition to a high frequency of accompanying infections, organs involved are various, and causative organisms are very diverse, including opportunistic pathogens, indicating the difficulty in treatment of the disease.

\section{Measures against the Infectability}

It is clear that only administration of massive dosages of antibiotics is not sufficient to combat infectious states in immunological diseases. Other measures are good nutrition of patients, clean environment with isolated and germ-free wards for patients, and parenteral infusion of human gammaglobulin preparations concomitantly with antibiotics.

In this paper, use of immunomodulators for infectability will be discussed. ${ }^{1-6)}$ The drugs we are dealing with are: 1) D-penicillamine, 2) Levamisole, 3) N-(2-carboxyphenyl)-4-chloroanthranilic acid disodium salt (CCA), 4) $\mathrm{N}$-(2mercapto-2-methylpropanoyl)-L-cystein (DE019) (SA 96), 5) Traxanox (Trax) (Y-12.141), 6) Mecobalamin, and 7) Inosiplex (MND-19). It has been proved that these drugs modify immune functions, and return depressed immune functions to normal levels in patients. They are known to activate functions of macrophages and lymphocytes, in particular those of suppressor $\mathrm{T}$ cells. In addition, they have been found useful in prevention and treatment of SLE-like disease in New Zealand mice and experimental adjuvant disease in rats. Therefore, these agents are used in immunological diseases, e.g. RA and SLE, with beneficial effects, as well as in immunodeficiency, malignancy, and severe infections.

Recently, in my laboratory, several immunomodulators were examined for phagocytic activity against macrophages obtained from peritoneal exudates after stimulation of phosphate buffer solution in BALB/c mice (Table 1). These cells were mixed with Candida parapsilosis in vitro, and candidacidal activity was determined as MNMK (Maximal number of microbes killed) in vitro.

Solutions of SA 96 in various concentrations were added, and dose-dependent activation of phagocytosis to Candida was exhibited, although suppression was seen at a concentration of $100 \mu \mathrm{g} /$ ml (Table 2). Next, according to the method shown in Table 3, various immunomodulators in various concentrations were examined for phagocytic activity of peritoneal cells against Candida in vivo. As a result, SA 96, D-penicillamine, Levamisole and E. coli lipopolysaccharides was found to activate phagocytosis of peritoneal cells against Candida, while CCA, Traxanox and

Table 1. Outline of the technique for assessing the candidacidal activity of peritoneal exudate cells

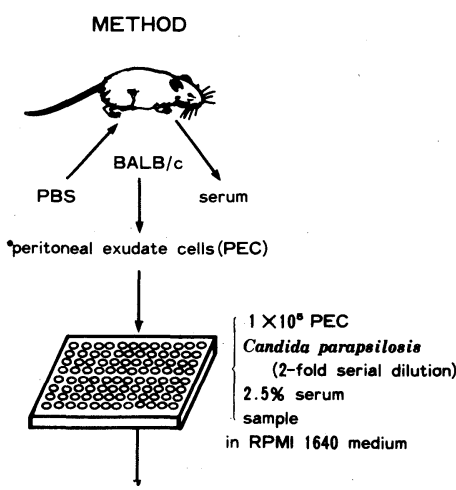

outgrowth of candida cells in wells was determined

\begin{tabular}{|c|c|c|c|c|c|c|c|c|c|c|c|c|c|}
\hline \multirow{2}{*}{$\begin{array}{c}\text { serum } \\
(\boldsymbol{(})\end{array}$} & \multicolumn{11}{|c|}{ microbial dilution } & \multicolumn{2}{|c|}{ candidacidal activity } \\
\hline & $2^{\circ}$ & $2^{-1}$ & $2^{-2}$ & $2^{-3}$ & $2^{-4}$ & $2^{-6}$ & $2^{-\bullet}$ & $2^{-7}$ & $2^{-\bullet}$ & $2^{-\bullet}$ & $2^{-10}$ & MNMK & $\begin{array}{c}\text { increase } \\
(n-\text {-fold })\end{array}$ \\
\hline 0 & + & + & + & + & + & + & - & - & - & - & - & 41.6 & \\
\hline 1.0 & + & + & $\begin{array}{llll}+ & -1 & -1\end{array}$ & \pm & - & - & - & - & - & - & - & 166.4 & 4 \\
\hline 2.5 & + & + & + & \pm & - & - & - & - & - & - & - & 166.4 & 4 \\
\hline 5.0 & + & + & + & \pm & - & - & - & - & - & - & - & 166.4 & 4 \\
\hline
\end{tabular}


Table 2. Effects of SA 96 on the candidacidal activity of normal peritoneal exudate cells

\begin{tabular}{lrcc}
\hline & $\begin{array}{c}\text { Conc. } \\
(\mu \mathrm{g} / \mathrm{ml})\end{array}$ & \multicolumn{2}{c}{ Candidacidal activity } \\
\cline { 3 - 4 } & & 188.8 & $\begin{array}{c}\text { Increase } \\
\text { (n-fold) }\end{array}$ \\
\hline Control & 0.1 & 377.6 & 2 \\
SA 96 & 1.0 & 755.2 & 4 \\
& 10.0 & 755.2 & 4 \\
& 100.0 & 47.2 & 0.25 \\
\hline
\end{tabular}

Table 3. Candidacidal activity of peritoneal exudate celis from mice treated with various immunomodulators

\begin{tabular}{|c|c|c|c|}
\hline \multicolumn{2}{|r|}{ Treatment } & \multicolumn{2}{|c|}{ Candidacidal activity } \\
\hline & & MNMK & $\begin{array}{r}\text { Increase } \\
\text { (n-fold) }\end{array}$ \\
\hline \multicolumn{2}{|c|}{ Control } & 136.0 & \\
\hline SA 96 & $25 \mathrm{mg} / \mathrm{kg}$ p.o. for 2 days & 1088.0 & 8 \\
\hline D-Pc & $50 \mathrm{mg} / \mathrm{kg}$ p.o. for 2 days & 1088.0 & 8 \\
\hline LMS & $25 \mathrm{mg} / \mathrm{kg}$ p.o. for 2 days & 544.0 & 4 \\
\hline CCA & $50 \mathrm{mg} / \mathrm{kg}$ p.o. for 2 days & 136.0 & 1 \\
\hline Trax & $30 \mathrm{mg} / \mathrm{kg}$ p.o. for 2 days & 136.0 & 1 \\
\hline $\mathrm{HC}$ & $50 \mathrm{mg} / \mathrm{kg}$ p.o. for 2 days & 272.0 & 2 \\
\hline LPS & $1.5 \mathrm{mg} / \mathrm{kg}$ i.p. & 4325.0 & 32 \\
\hline
\end{tabular}

LPS was administered on day 0 and candidacidal activity was assayed on day 2; other drugs were administered two times on day 0 and day 1 and the assay was performed on day 4 .

hydrocortisone had no such effect. In clinical studies, Traxanox, $540 \mathrm{mgm}$ per day orally, was given to patients with SLE for one month. During this period, the dosage of corticosteroids and other drugs administered was not changed. Four out of 5 patients showed positive bacteria in the urine in microscopic examinations at the start. At the end of the trial, only one remained positive, and another 3 cases showed negative urine findings, indicating that the use of Traxanox might suppress urinary tract infections in SLE. Further studies will be needed, but immunomodulators can be utilized in microbial infections occurring in patients with abnormal immune functions, or in a state of infectability, with or without the use of antibiotics.

\section{SUMMARY AND CONCLUSION}

Patients with connective tissue diseases such as SLE are liable to infections, which could become a prevailing cause of death in these disorders. Prevention and treatment of infectability in these diseases are becoming more and more important in clinical medicine. In this paper, it was suggested that immunomodulators might be utilized for this purpose with or without antibiotics.

\section{REFERENCES}

1) Abe G, Nakazawa T, Shiokawa Y: D-penicillamine in the modulation of immune responses in mice. Int J Clin Pharm Res 1, 225-232, 1981.

2) Abe C, Shiokawa Y, Ohishi T, Hata S, Takagaki Y: The effect of N-(2-carboxyphenyl)-4-chloroanthranilic acid disodium salt (CCA) on spontaneous autoimmune disorders in MRL/1 mice. Ryumachi 21 (Suppl), 165-170, 1981.

3) Yamamoto I, Ohmori H, Okimura T: SA 96: an immunopharmacological profile in vitro. Jap J Inflammation 1, 133-138, 1980.

4) Terasawa M, Imayoshi T, Goto K, Maruyama Y: Effect of a new benzopyranopyridine derivative Y-12141. Jap J Inflammation 1, 127-132, 1980.

5) Sakane T, Honda M, Taniguchi Y, Kotani H: Immunomodulating activity of methyl-B $\mathrm{B}_{12}$ for the in vitro human $\mathrm{T}$ cell functions. Jap $\mathrm{J}$ Allergol 30, 244-251, 1981.

6) Hadden JW, Lopez C, O'Reilly RJ: Levamisole and Inosiplex: Antiviral agents with immunopotentiating action. Ann N Y Acad Sci 286, 139-152, 1977. 